\title{
Editorial
}

\section{Community singing: what does that have to do with health?}

\author{
Jing Sun, Nicholas J. Buys and Joav Merrick
}

Arts and health is an emerging multidisciplinary area of research, policy, and practice. It includes a variety of ways in which the arts contribute to health, well-being, and health care practice across a range of contexts (1). We believe that the time has arrived to conduct a project where art and health are aligned with concepts drawn from performing arts (music and singing, and singing performance festivals) and Aboriginal community cultural development as a practice to initiate change. Through an innovative arts-based "resilience-building" intervention approach derived from these ideas, our project will address how Aboriginal and Torres Strait Islander people can obtain support for their mental health issues. Furthermore, it will build on a substantial record in designing and implementing innovative interdisciplinary arts-based interventions to support people with mental health issues (2), drawing on existing international collaborations in similar settings $(2,3)$.

The value of the arts in addressing significant social issues (4) and improving the lives across the whole of the lifespan (5) has been increasingly recognized, and particularly the value of live music and musical participation for people with mental illness (4). Singing is an activity that has been shown to carry benefits for health and well-being across the lifespan and with people of diverse social backgrounds and health status (6). Extant Australian and international research shows that participatory community singing is a powerful means to enhance participation, social engagement, and interpersonal interaction with others and brings a wide range of benefits to participants for social, emotional, mental, and spiritual involvement (7). Studies focused on the impact of participation on mental health can be placed in a broader context of a participatory model of health promotion, in which the focus is on the strategies and process of participation as well as the inter-relationships between participation in meaningful activity, emotional states, and mental health outcomes.

Community singing is part of Aboriginal and Torres Strait Islander culture and has long been associated with social gatherings, ceremonies, and festivals. These social activities have been found to help raise self-esteem and sense of social connectedness of Aboriginal and Torres Strait Islander people (8).

A community-based participatory research approach in these communities can foster a sense of self-determination, creates greater commitment, can ultimately improve selfesteem, increase a sense of belonging, and protects against depression and anxiety (9). The process of participation, including collaboration with service providers and local community members to create supportive environments and social support, codetermination of action plans, and collective actions, has been shown to increase resilience and mental health through development of positive attitudes, self-esteem and community pride (8), and access to community-controlled health services. This approach overcomes the shortcomings of most health promotion interventions that primarily rely upon educational and information-based strategies (10). Participatory community singing activities may impact on mental health through both enhancement of positive emotional states and via a sense of resilience development and social connectedness (11). Such activity also has been shown in our study to diminish loneliness, increase the sense of mastery and self-esteem, reduce the risk of depression, and decrease the number of doctor visits and medication use (11). It therefore has strong potential in engaging Aboriginal and Torres Strait Islander people in managing mental health issues.

\section{References}

1. Clift S, Hancox G. The significance of choral singing for sustaining psychological wellbeing: findings from a survey of choristers in England, Germany and Australia. Community Music Activity, 2008.

2. Clift S, Hancox G, Morrison I, Hess B, Kreutz G, et al. Choral singing and psychological wellbeing: quantitative and qualitative findings from English choirs in a cross-national survey. J Appl Arts Health 2010;1:19-34.

3. Clift S, Hancox G, Morrison I, Hess B, Kreutz G, et al. editors. Choral singing and psychological wellbeing: findings from English choirs in a cross-national survey using the WHOQoL-BREF. Proceedings of the International Symposium on Performance Science, Porto, 2007.

4. Skingley A, Clift MS, Coulton SP, Rodriguez J. The effectiveness and cost-effectiveness of a participative community singing programme as a health promotion initiative for older people: protocol for a randomised controlled trial. BMC Public Health. 2011;11:142.

5. Batt-Rawden KB, Tellnes G. Nature-culture-health activities as a method of rehabilitation: an evaluation of participants' health, quality of life and function. Int J Rehabil Res 2005;28:175-80.

6. Bamford A. Making singing for health happen. Canterbury: Canterbury Christ Church University, 2006.

7. Cohen GD, Perlstein S, Chapline J, Kelly J, Firth KM, et al. The impact of professionally conducted cultural programs on the physical health, mental health, and social functioning of older adults. Gerontologist 2006;46:726-34.

8. Stacey K, Keller N, Gibson B, Johnson R, Jury L, et al. Promoting mental health and well-being in Aboriginal contexts: successful elements of suicide prevention work. Health Promot J Austr 2007;18:247-54. 
9. Wall M, Hayes R, Moore D, Petticrew M, Clow A, et al. Evaluation of community level interventions to address social and structural determinants of health: a cluster randomised controlled trial. BMC Public Health 2009;9:1-11.

10. De Bock F, Fischer JE, Hoffmann K, Renz-Polster H. A participatory parent-focused intervention promoting physical activity in preschools: design of a cluster-randomized trial. BMC Public Health 2010;10:49.

11. Sun J, Buys N. Participatory community singing and mental health promotion in Aboriginal and Torres Strait Islander people. Mental Health Tech 2012, in press.

Jing $\operatorname{Sun}^{1,2, *}$

Nicholas J. Buys 2,3

Joav Merrick ${ }^{4-7}$

${ }^{1}$ School of Public Health and Griffith Health Institute, Griffith University, Logan Campus, Meadowbrook, Australia

${ }^{2}$ School of Public Health, Peking University, P R China
${ }^{3}$ School of Human Services and Social Work and Griffith Health Institute, Griffith University, Gold Coast Campus, Parkland, Australia

${ }^{4}$ National Institute of Child Health and Human Development, Jerusalem, Israel

${ }^{5}$ Division of Pediatrics, Hadassah Hebrew University Medical Centers, Mt Scopus Campus, Jerusalem, Israel

${ }^{6}$ Office of the Medical Director, Health Services, Division for Intellectual and Developmental Disabilities, Ministry of Social Affairs and Social Services, Jerusalem, Israel

${ }^{7}$ Kentucky Children's Hospital, University of Kentucky, Lexington, USA

*Corresponding author: Jing Sun, PhD, School of Public Health and Griffith Health Institute, Griffith University, Logan Campus, Meadowbrook, Q4131 Australia

E-mail: j.sun@griffith.edu.au 